Revue de droit comparé du travail et de la sécurité sociale

3 | 2017

Le travail dans l'économie informelle, un défi pour le droit social

\title{
Economie informelle, protection sociale et transition vers l'économie formelle : les termes d'un débat
}

Jacques Charmes

\section{OpenEdition}

Journals

Édition électronique

URL : https://journals.openedition.org/rdctss/307

DOI : $10.4000 /$ rdctss.307

ISSN : 2262-9815

Éditeur

Centre de droit comparé du travail et de la sécurité sociale

Édition imprimée

Date de publication : 1 septembre 2017

Pagination : 10-17

ISSN : 2117-4350

Référence électronique

Jacques Charmes, «Economie informelle, protection sociale et transition vers l'économie formelle les termes d'un débat », Revue de droit comparé du travail et de la sécurité sociale [En ligne], 3 | 2017, mis en ligne le 01 novembre 2018, consulté le 04 décembre 2021. URL : http://journals.openedition.org/ rdctss/307; DOI : https://doi.org/10.4000/rdctss.307

\section{(c)}

Revue de droit comparé du travail et de la sécurité sociale est mise à disposition selon les termes de la Licence Creative Commons Attribution - Pas d'Utilisation Commerciale - Pas de Modification 4.0 International. 


\section{ÉCONOMIE INFORMELLE, PROTECTION SOCIALE ET TRANSITION VERS L'ÉCONOMIE FORMELLE : \\ LES TERMES D'UN DÉBAT}

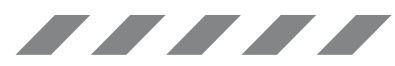

\section{ABSTRACT}

The concepts of informal sector, informal employment and informal economy continue to be the subject of many misunderstandings despite progress made in their international definitions. This is why it is always necessary and useful to recall the terms of these international definitions which have enabled more precise measurements of these phenomena and have also made it possible to adopt recommendations with a view to achieving a consensual transition from the informal economy to the formal economy. Such a transition involves, among other things, the universalization of social protection covering the basic risks (health, maternity, old age, etc.) that is enshrined in the Sustainable Development Goals.

KEYWORDS : Informal employment, informal economy, informal sector, social protection.

\section{RÉSUMÉ}

Les concepts de secteur informel, d'emploi informel et d'économie informelle continuent à faire l'objet de nombreux contresens, malgré les progrès enregistrés dans leurs définitions internationales. C'est pourquoi il est toujours nécessaire et utile de rappeler les termes de ces définitions internationales qui ont permis des mesures plus précises de ces phénomènes et ont également permis l'adoption de recommandations en vue d'assurer de manière consensuelle la transition de l'économie informelle vers l'économie formelle. Une telle transition passe entre autres par l'universalisation d'une protection sociale assurant la couverture des risques fondamentaux Isanté, maternité, retraite ...) qui est inscrite dans les Objectifs du Développement durable.

\section{MOTS CLÉS : Emploi informel, économie informelle, secteur informel, protection sociale.}


algré quelque 45 ans de recul - puisque c'est en 1971-72 que fut pour la première fois utilisé le terme - le concept d'économie informelle continue à faire l'objet de grandes confusions et contresens tant de la part des politiques que des économistes. Le plus courant de ces contresens est l'assimilation de l'économie informelle à l'économie souterraine, l'économie de l'ombre (« shadoweconomy») et en définitive l'économie illégale. Si l'on revient pourtant aux origines du concept, on retiendra que ces activités « ne sont pas nécessairement réalisées avec l'intention délibérée de se soustraire au paiement des impôts ou des cotisations de sécurité sociale, ou d'enfreindre la législation du travail, d'autres législations, ou d'autres dispositions administratives » pour reprendre les termes de la première définition qui fut adoptée en 1993. Et, en effet, les travailleurs de l'économie informelle n'opèrent pas au clair de lune (« moonligting») mais en plein soleil, au vu et au su de tout le monde. D'ailleurs, le terme Swahili utilisé pour les décrire au Kenya (pays où naquit le concept en 1971-72) est « Jua Kali» qui signifie « sous le soleil brûlant».

En réalité, plutôt qu'une volonté délibérée de ces acteurs de se soustraire aux législations en vigueur, c'est d'une certaine incapacité ou une certaine impuissance de l'État à faire appliquer ses propres réglementations qu'il conviendrait de parler, soit parce que celles-ci sont profondément inadaptées, ayant été calquées sur celles des anciens colonisateurs, soit parce que les processus de modernisation et d'industrialisation ne s'étant pas enclenchés comme le prévoyait la vision optimiste du développement, l'adaptation progressive de l'économie réelle à ces réglementations n'est pas intervenue aussi rapidement que souhaité. D'où en serait résultée une certaine tolérance vis-à-vis de ces activités qui, par ailleurs, nourrit une corruption rampante leur permettant de continuer à s'exercer.

Néanmoins, la constante progression des emplois informels n'a pas manqué de renvoyer aux pouvoirs publics l'image de leur impuissance, d'autant plus ravageuse que la dynamique de l'économie informelle avait pour contrepoint la stagnation ou le blocage d'un secteur formel dominé par un secteur public hypertrophié. Cependant, le secteur privé formel, étriqué, ne manquait pas de sensibiliser l'administration à cette concurrence déloyale qui aurait été la cause de ses difficultés et certes une fraction des activités informelles est désormais constituée d'unités économiques manifestant la volonté d'échapper aux législations en vigueur et est venue se greffer sur le secteur. Rares sont les pays qui admettent ou reconnaissent officiellement l'existence d'une économie informelle qui mériterait qu'on lui dédie des politiques compréhensives. Ce sont des termes plus positifs et moins chargés qui sont alors mis en avant : travail indépendant, micro-entreprises, artisanat et petits métiers, activités génératrices de revenus, etc.

Il n'en reste pas moins que l'on parle d'emplois présentant toutes les caractéristiques de la précarité. L'Organisation Internationale du Travail (OIT) qui, en un temps où le chômage et le sous emploi amorçaient leur lancinant et durable essor, avait mis l'accent sur les aspects positifs liés à ces activités et à leur dynamique, depuis le rapport fondateur sur l'emploi au Kenya ${ }^{01}$, puis sur la promotion de l'emploi indépendant ${ }^{02}$, en vint ensuite à en poser la problématique sous la forme

01 ILO, Employment, Incomes and Equality. A Strategy for Increasing Productive Employment in Kenya. ILO, Geneva, 1972.

02 BIT, Promotion de l'emploi indépendant, Rapport du Directeur Général, Conférence Internationale du Travail, $77^{\mathrm{e}}$ session, Genève, 1990 
d'un dilemme ${ }^{03}$, puis à définir les termes du travail décent ${ }^{04}$, à poser le principe des socles de protection sociale $e^{05}$, pour finalement, dans le cadre de sa vocation tripartite lorganisant le dialogue et le consensus entre gouvernements, employeurs et travailleurs), adopter à l'unanimité en 2015 une recommandation en vue de favoriser la transition de l'économie informelle à l'économie formelle ${ }^{06}$. Or l'universalisation de la protection sociale, inscrite comme troisième cible du premier des Objectifs de Développement durable (ODD) et réitérée parmi les cibles de plusieurs autres ODD (santé, égalité entre les sexes, réduction des inégalités) est l'une des voies que doit prendre le processus de transition vers l'économie formelle.

Après avoir rappelé les définitions de l'économie informelle et de ses principales composantes (I), nous examinerons les potentialités d'extension de la protection sociale à ces activités (II) en replaçant ensuite cette démarche dans le cadre plus vaste des stratégies de transition de l'économie informelle vers l'économie formelle (III).

\section{I - Définitions de l'économie informelle}

Dès l'origine, deux conceptions se sont affrontées ou plutôt complétées. La première - dans la foulée du rapport de l'OIT sur le Kenya ${ }^{07}$ - définit l'informalité par les caractéristiques de l'entreprise dans laquelle travaille la personne ; la seconde définit le phénomène - à la suite de Keith Hart ${ }^{08}$ qui parlait « d'opportunités informelles de revenus » - par les caractéristiques de l'emploi occupé par la personne.

C'est la première conception qui a tout d'abord prévalu et a débouché sur une première définition de l'emploi dans le secteur informel en 1993. La 15e Conférence Internationale des Statisticiens du Travail (CIST), en charge de la définition des concepts de population active, adopte en 1993 une Recommandation en ce sens ${ }^{09}$. Celle-ci rappelle que le secteur informel consiste en un ensemble d'unités produisant des biens et des services en vue principalement de créer des emplois et des revenus pour les personnes concernées, des unités à faible niveau d'organisation, opérant avec peu ou pas de division entre travail et capital, sur une petite échelle, et avec des relations d'emploi fondées, lorsqu'elles existent, sur des liens de parenté ou des relations personnelles ou sociales plutôt que sur des accords contractuels comportant des garanties. Plus concrètement, il s'agit d'un ensemble d'unités de production constituées en entreprises individuelles (par opposition aux sociétés et quasi-sociétés qui tiennent une comptabilité

03 BIT (1991), Le dilemme du secteur non structuré, Rapport du Directeur Général, Conférence Internationale du Travail, 78 ème session, Genève, 1991

04 BIT (1999), Travail Décent, Rapport du Directeur Général, Conférence Internationale du Travail, 87ème session, Genève, 1999. BIT, Travail décent et économie informelle, Conférence Internationale du Travail, $90^{\circ}$ session, Genève, 2002

05 BIT, Recommandation 202 - Recommandation sur les socles de protection sociale, adoptée par la Conférence à sa $101^{\mathrm{ème}}$ session, Genève, 14 juin 2012

$06 \mathrm{BIT}, \mathrm{La}$ transition de l'économie informelle vers l'économie formelle, Conférence Internationale du Travail, Rapport V (1), 103e session, 2014. BIT, Recommandation 204 - Recommandation concernant la transition de l'économie informelle vers l'économie formelle, adoptée par la Conférence à sa $104^{\circ}$ session, Genève, 12 juin 2015.

07 ILO, Employment, Incomes and Equality. A Strategy for Increasing Productive Employment in Kenya, ILO, 1972, Geneva.

08 Hart, K., «Informal Income Opportunities and Urban Employment in Ghana », Journal of Modern African Studies, 1971, vol. II

09 BIT, Statistiques de l'emploi dans le secteur informel, Rapport pour la XVe Conférence Internationale des Statisticiens du Travail, Genève 19-28 Janvier 1993. BIT, Rapport de la Conférence, Rapport pour la XV Conférence Internationale des Statisticiens du Travail, Genève 19-28 Janvier 1993, BIT 
complète, ou aux associations) et, en tant que telles, appartenant au secteur institutionnel des ménages dans les comptes nationaux. On distingue les travailleurs indépendants qui peuvent employer des travailleurs familiaux et des salariés de façon occasionnelle, et les employeurs informels qui emploient un ou plusieurs salariés de façon continue, en dessous d'un seuil de taille (généralement fixé à 5), ou/et sans les enregistrer, ou/et sans être enregistrés en tant qu'entreprise.

Cette définition a été appliquée dans de nombreuses enquêtes à partir de 1993 et a suscité des politiques d'appui aux travailleurs indépendants et aux micro-entreprises dans de nombreux pays en développement.

Toutefois l'adoption de cette définition n'a pas rendu caduque pour autant la conception de l'informalité par les caractéristiques de l'emploi, d'autant plus que la mondialisation se traduisait par une expansion des processus de sous-traitance, de développement du travail à domicile, d'externalisation et de précarisation des emplois, de sorte que la définition de 1993 apparaissait ne couvrir qu'une frange certes importante mais incomplète du processus d'informalisation.

C'est pourquoi la $17^{\mathrm{e}} \mathrm{CIST}^{10}$ va proposer en 2003 des lignes directrices pour définir l'emploi informel. L'emploi informel qualifie les travailleurs et spécifiquement les salariés « dont la relation d'emploi n'est pas soumise, de par la loi ou en pratique, à la législation nationale du travail, l'impôt sur le revenu, la protection sociale ou le droit à certains avantages liés à l'emploi (par exemple, préavis en cas de licenciement, indemnité de licenciement, congés payés annuels ou congés de maladie payés, etc.) parce que les salariés ou leurs emplois ne sont pas déclarés; les emplois sont occasionnels ou de courte durée; le temps de travail ou le salaire n'atteignent pas un certain seuil (...) ; le lieu de travail du salarié se situe en dehors des locaux de l'entreprise de l'employeur (travailleurs extérieurs à l'établissement et ne bénéficiant pas d'un contrat de travail) ; la législation du travail n'est pas appliquée, respectée ou observée pour tout autre motif ». Couvrant également les travailleurs non salariés, cette définition se résume - et prend la forme de cette question dans les enquêtes - à l'absence de protection sociale ou au non paiement de contributions sociales (spécialement pour la couverture santé).

Loin de rendre obsolète la définition de 1993 de l'emploi dans le secteur informel, la définition de l'emploi informel de 2003 la complète.

En définitive, les deux définitions ne sont pas mutuellement exclusives et se recouvrent en partie, de sorte que ce qu'il est convenu d'appeler l'emploi dans l'économie informelle comprend plusieurs composantes : 1) l'emploi dans le secteur informel, 2) l'emploi informel dans le secteur formel, 3) les travailleurs domestiques informels, et enfin 4) les personnes engagées dans la production de biens pour usage final propre de leurs ménages. Bien que non assujettie au droit jusqu'à présent, cette dernière catégorie est susceptible d'être bénéficiaire de droits au regard de la protection sociale en particulier. Les aspects juridiques relatifs à l'économie informelle et à sa transition vers l'économie formelle présentent donc un double aspect : celui de l'entreprise et celui du travailleur.

On l'aura compris, la définition de l'économie informelle, au contraire de la « shadow economy», est celle qui peut se traduire en nombre d'emplois. Il y a pourtant un autre aspect de l'informalité qu'il est nécessaire de prendre en compte lorsqu'on s'intéresse aux droits des travailleurs : c'est celui de ses marges. Ainsi en est-il d'un certain nombre de salariés immatriculés à la sécurité sociale - donc formels - qui ne le sont qu'au simple niveau du salaire minimum légal. Le contrat tacite qui permet de réduire les cotisations tant des employeurs que des travailleurs est qu'une partie du salaire est

10 BIT, XVIle Conférence Internationale des Statisticiens du Travail, Rapport Général 1, Genève, 2003. BIT, Rapport de la XVII Conférence Internationale des Statisticiens du Travail, Genève, 2003. 
versée de la main à la main, les prestations étant évidemment réduites d'autant, qu'il s'agisse des congés maladie ou des droits à la retraite en particulier. Une comparaison systématique des statistiques salariales tirées des répertoires de sécurité sociale (malheureusement rarement disponibles dans les pays en développement) avec les statistiques tirées des enquêtes auprès des ménages permet d'en mesurer l'ampleur.

\section{II - Quelle protection sociale pour les acteurs de l'économie informelle?}

Sur la base des définitions précédentes, on sait désormais que l'emploi dans l'économie informelle représente jusqu'à $73 \%$ de la population occupée non agricole en Afrique subsaharienne, $65 \%$ en Asie, $57 \%$ en Amérique latine, $48 \%$ en Afrique du Nord et $25 \%$ dans les économies en transition d'Europe de l'Est et d'Asie centrale ${ }^{11}$. Ces chiffres seraient encore plus élevés si l'on y incluait l'agriculture puisque dans le plus grand nombre des pays en développement, la quasi-totalité des emplois agricoles sont informels. La contribution du secteur informel au PIB représente pour sa part quelque $55 \%$ en Afrique sub-Saharienne let $25 \%$ du PIB non agricole), $32 \%$ en Afrique du Nord, 30\% en Amérique latine et $42 \%$ en Inde dans les années $2010^{12}$. Une autre façon de lire ces chiffres est de considérer que ces populations et leurs dépendants ne sont couverts par aucune protection sociale autre que ce qu'il est convenu d'appeler les filets sociaux, c'est-à-dire une assistance sociale se limitant à la distribution de revenus minimums ou de rations de nourriture, souvent en contrepartie d'un travail, si toutefois ces filets sociaux les atteignent.

Si l'on préfère ignorer les termes d'éradication, d'élimination, de lutte qu'a inspirés l'économie informelle aux pouvoirs publics, sensibles aux arguments de concurrence déloyale, c'est sa fiscalisation qui a fait l'objet des plus nombreuses réflexions : si ce secteur représentait entre le tiers et le quart du PIB, ne s'agissait-il pas d'une base taxable importante en un temps où celle-ci se faisait de plus en plus évanescente pour des trésors publics en mal de recettes budgétaires ? Mais le prélèvement de l'impôt, à part la satisfaction d' « être en règle », ne se traduit pas par des contreparties tangibles et immédiates pour des opérateurs qui généralement ont peu bénéficié et bénéficient peu de l'accès aux services publics, qu'il s'agisse de l'éducation, de la santé, sans même parler des services de facilitation des activités économiques /crédits, marchés, etc.). Par ailleurs, pour le fisc, le coût de recouvrement reste prohibitif. Dès lors, la réflexion s'étendit à l'ensemble des prélèvements obligatoires et aux contributions sociales, en particulier. Des systèmes fiscaux adaptés, comme les systèmes Monotax en Argentine ou Simples au Brésil, fixaient le montant forfaitaire de l'impôt en fonction de critères simples tels que la consommation d'électricité ou la superficie du local (parallèlement et alternativement au chiffre d'affaires) et surtout le montant était déclaré libératoire de toutes les autres contributions dues : en particulier une certaine proportion de l'impôt versé était automatiquement destinée à alimenter le système de sécurité sociale.

Dans d'autres régions où les systèmes de sécurité sociale sont plus fragiles et souvent faiblement étendus au-delà du secteur public, comme en Afrique sub-Saharienne, l'extension de la protection sociale à l'économie informelle s'appuie sur un système mutualiste, centré autour de corps de métiers : agriculteurs, artisans, commerçants. Des mutuelles ou des coopératives

11 J. Charmes, « The informal economy worldwide: trends and characteristics Margin », The Journal of Applied Economic Research, 6, 2, 2012, p. 103-132. ILO, Statistical Update on Employment in the informal Economy, Geneva, 2011.

12 J. Charmes, ibid. 
sont mises en place, reprenant souvent des formes d'associations communautaires anciennes, qui regroupent des adhérents bénéficiaires. Des institutions officielles (publiques ou privées, telles que des ONG dépositaires de la gestion de projets financés par des donneurs extérieurs) les chapeautent, garantissant la bonne gestion et répartition des fonds collectés. Les prélèvements sociaux s'adossent à des dépôts d'épargne destinés à bénéficier de crédits. Un système original consiste à adosser les cotisations (à une couverture santé ou/et retraite) au remboursement d'un prêt consenti pour l'achat d'équipement, ou à l'achat d'un intrant indispensable lengrais pour les agriculteurs, carburant pour les transporteurs), l'idée étant de rendre indolore la cotisation régulière. La Délégation à l'Organisation du Secteur Informel ${ }^{13}$ au Togo a ainsi constitué plusieurs mutuelles lagriculteurs, pêcheurs et éleveurs, conducteurs de taxis-motos, artisans et commerçants, artistes et journalistes) qui assurent une couverture santé à plusieurs dizaines de milliers de bénéficiaires. En Côte d'Ivoire, des coopératives d'artisans (menuisiers, couturières, sculpteurs, potières) ont rejoint des mutuelles de santé par l'intermédiation d'un projet européen conduit par l'ONG italienne AVSI. Au Ghana, c'est encore un projet européen (PlanetFinance) qui a sensibilisé et organisé les femmes travaillant les noix de karité, pour en faire du beurre ou de la crème entrant dans la fabrication de produits cosmétiques haut de gamme, afin que celles-ci puissent cotiser au National Health Insurance System (NHIS). Un volet essentiel du projet a consisté à appuyer le NHIS lui-même pour l'amener à aller au-devant de ces femmes dans les régions, à se mettre à l'écoute de leurs besoins, à entendre leurs doléances sur le fonctionnement courant du système, à recueillir leurs cotisations et mettre à jour leurs cartes. Ainsi était mis en exergue l'aspect « quérable » (par l'institution) de la cotisation par rapport à la pratique « portable » (par l'assujetti ou l'assuré), une inversion des normes qui se révèle essentielle pour enclencher une dynamique vertueuse de l'administration visà-vis de l'économie informelle qui prétend ne rien lui devoir ni ne rien en attendre. Un récent rapport du Réseau de Recherche RNSF (Research Network Support Facility ${ }^{14}$ ) présente un inventaire de la diversité des bonnes pratiques dans ces domaines ${ }^{15}$.

L'économie sociale et solidaire (ESS) trouve ici un formidable domaine où se déployer à condition de convaincre des populations parfois échaudées par des expériences malheureuses. De fait, l'ESS peut être un acteur pivot dans la transition de l'économie informelle vers l'économie formelle.

\section{III - Vers une stratégie de transition de l'économie informelle vers l'économie formelle}

Lorsqu'en 2014 la Conférence Internationale du Travail s'empare du sujet de la transition, de par ses statuts et sa composition tripartite, elle se doit de concilier les points de vue a prioridivergents des gouvernements, des employeurs et des travailleurs à l'égard d'un phénomène qu'ils ne maîtrisent guère. Les gouvernements sont simultanément favorables à la répression lils doivent faire respecter leurs réglementations) et à la tolérance (l'auto-emploi est une solution à l'inefficacité des politiques de création d'emploil ; les employeurs sont favorables à l'éradication d'activités qui leur font une concurrence déloyale sans reconnaître que ces activités leur permettent de maintenir des taux de

13 DOSI, De la mutualisation à la formalisation. Les actions de la Délégation à l'Organisation du Secteur Informel (DOSI), Lomé, 2015, 18p.

14 The Research Network Support Facility (RNSF) est un projet financé par l'Union Européenne pour l'appui à l'amélioration des modes de vie des populations dépendantes de l'économie informelle et à l'inclusion sociale des personnes marginalisées et vulnérables. Le projet s'appuie sur l'analyse des bonnes pratiques et des leçons tirées de projets de développement passés et en cours. https://europa.eu/capacity4dev/iesf/dashboard

15 RNSF, Extending coverage: Social protection and the informal economy. Experiences and ideas from researchers and practitioners, Research, Network and Support Facility, ARS Progetti, Rome; Lattanzio Advisory, Milan; and AGRER, Brussels, European Union, 2017. 
salaire bas ; quant aux syndicats de travailleurs, ils reconnaissent que ces travailleurs - qui ne sont pas adhérents à leurs organisations - ont le droit de bénéficier des normes fondamentales du travail définies dans le cadre de l'OIT.

Après une première discussion du sujet en 2014, c'est en juin 2015 qu'est adoptée la Recommandation $204^{16}$, qui va définir le cadre des actions à venir en matière de politique d'emploi dans les pays en développement.

Reconnaissant que la plupart des individus n'entrent pas dans l'économie informelle par choix mais bien par nécessité, faute de trouver un emploi dans l'économie formelle, la Recommandation se fixe trois objectifs : faciliter la transition des travailleurs et des unités économiques vers l'économie formelle; promouvoir la création d'entreprises et d'emplois décents, leur préservation et leur pérennité ; prévenir l'informalisation des emplois de l'économie formelle. Une telle diversité des situations implique une égale diversité des stratégies et politiques à mettre en œuvre et la nécessité de coordination qui en résulte.

L'un des principes de base est la nécessité d'adopter une approche équilibrée, combinant des mesures incitatives et des mesures correctives. Car la formalisation ne doit pas casser le potentiel entrepreneurial, sa créativité, son dynamisme, ses compétences et ses capacités d'innovation. Par ailleurs, il est nécessaire de sanctionner le contournement des législations fiscales, sociales et du travail par les entreprises qui ont en vue de sortir de l'économie formelle.

Les orientations concernant les micro-entreprises consistent à réduire les coûts d'enregistrement, la longueur des procédures, à améliorer l'accès aux services, en particulier à travers les technologies de l'information et de la communication ; à réduire les coûts de mise en conformité en simplifiant les modes de calcul et de paiement des impôts et des cotisations ; à faciliter l'accès aux marchés publics en adaptant les procédures, en instituant des quotas et en dispensant des formations adéquates. ; à améliorer l'accès à des services financiers inclusifs ; à améliorer l'accès à la formation à l'entrepreneuriat; et à améliorer l'accès à la protection sociale.

Concernant les travailleurs, les enjeux sont : la mise à disposition d'informations et d'assistances : le renforcement des capacités ; l'instauration de procédures efficaces et accessibles de plainte et de recours; l'application effective des sanctions administratives, civiles et pénales.

Un certain nombre de pays, tels Madagascar, le Burkina Faso ou encore le Togo sont en train de définir des stratégies de transition vers l'économie formelle sur la base des préconisations de la Recommandation 204. Nombreux sont encore les pays à avoir pris des mesures dans plusieurs des domaines précédemment cités (sans toutefois en viser la coordination), notamment celui de la protection sociale.

16 BIT, Recommandation 204 - Recommandation concernant la transition de l'économie informelle vers l'économie formelle, adoptée par la Conférence à sa 104e session, Genève, 12 juin 2015. 


\section{Conclusion}

Les politiques de transition de l'économie informelle vers l'économie formelle mettent en jeu de nombreux aspects des politiques publiques (taxation, création d'entreprises, formation, protection socialel et des législations correspondantes, notamment sociales et du travail. Sous l'impulsion des nouveaux engagements de la communauté internationale (Objectifs du Développement durable), l'universalisation de la couverture santé et plus généralement de la protection sociale est inscrite sur l'agenda de la plupart des pays en développement et l'adoption de la Recommandation 204 en fait une dimension privilégiée de la stratégie de transition. Cela nous conduit à réfléchir aux évolutions nécessaires du droit social et du droit de la sécurité sociale : adapter les contributions sociales aux capacités de nouvelles populations et les prestations offertes à leurs besoins, répartir les rôles entre secteur public et secteur privé et aussi ce nouvel acteur qu'est l'économie sociale et solidaire, car les ambitieux objectifs des ODD ne pourront être atteints qu'en aidant les populations les plus vulnérables à s'organiser dans des contextes où la confiance en l'État et en ses institutions est profondément érodée.

\section{JACQUES CHARMES}

Directeur de recherche émérite IRD au Centre Population et Développement (CEPED : Université Paris Descartes-IRD), Professeur d'économie à l'Université de VersaillesSaint Quentin, Expert-recherche pour le projet de l'Union Européenne Recherche, Réseau et Mécanisme d'Appui (RNSF) pour l'« Appui à l'amélioration des moyens de subsistance des populations dépendantes de l'économie informelle et à l'inclusion sociale des personnes marginalisées et vulnérables ».

Parmi ses publications :

« The informal economy worldwide: trends and characteristics » in Margin-The Journal of Applied Economic Research, 6, 2, 2012, pp 103-132.

La jeunesse tunisienne et l'économie informelle, BIT, Tunis, 2015, p. 99.

$\sim$ J. Charmes et Nidhal Ben Cheikh, «Protection sociale et économie informelle en Tunisie, Les défis de la transition à l'économie formelle », CRES et Banque Africaine de Développement, Tunis, 2016, p. 92. 